\title{
Prosocialidad en Preescolares: Estudio de Teorías Subjetivas de Profesoras de Educación Inicial
}

\section{Prosociality in Preschoolers: Study of Subjective Theories of Early Education Teachers}

\author{
Ginella Cicardini ${ }^{1}$, Macarena Martínez ${ }^{1}$, Karen Ramírez ${ }^{1}$, David Cuadra-Martínez ${ }^{1}$, Pablo J. Castro-Carrasco ${ }^{2,3, *}$, \\ Nidia Slomp ${ }^{2}$ \\ ${ }^{1}$ Universidad de Atacama, Chile \\ ${ }^{2}$ Universidad de La Serena, Chile \\ ${ }^{1}$ Universidad Católica del Maule, Chile
}

\begin{abstract}
DESCRIPTORES:
Prosocial

Infante

Enseñanza

Aprendizaje

Educador

Teoría subjetiva

\section{RESUMEN:}

En edades tempranas no todos los infantes manifiestan apropiadamente una conducta prosocial, porque es necesario que interactúen con modelos y experiencias educativas sistemáticas ligadas a recibir ayuda de otros, sobre todo en el contexto de la educación inicial. Con el objetivo de describir e interpretar las teorías subjetivas acerca del desarrollo, enseñanza y aprendizaje del comportamiento prosocial que presentan educadoras de párvulo, se realizó un estudio descriptivo interpretativo, en base a un diseño de estudio de caso y metodología cualitativa. Mediante un muestreo intencional por conveniencia, se aplicaron entrevistas episódicas y grupos de discusión a 16 profesoras de educación inicial de una región del norte de Chile. Los datos se analizaron usando codificación temática y teoría fundamentada. Se encontraron teorías subjetivas esencialistas de la naturaleza humana, la infancia y la prosocialidad que sustentan las explicaciones que las educadoras poseen acerca de cómo se desarrolla, enseña y aprende la prosocialidad en los infantes, además de prácticas pedagógicas poco sistemáticas que se regulan en función de estas teorías subjetivas. En la discusión se analizan estos resultados a partir de la literatura existente en prosocialidad, proponiendo nuevos focos de investigación en el tema y algunas sugerencias que podrían impulsar la educación en prosocialidad.
\end{abstract}

\section{KEYWORDS:}

Prosocial

Infant

Teaching

Learning

Educator

Subjective theory

\section{ABSTRACT:}

Infants would need to interact with systematic educational models, and experiences linked to receiving help from others, especially in the context of early education. With the aim of describing and interpreting preschool teachers' subjective theories about the development, teaching, and learning of prosocial behavior, we conducted an interpretative and descriptive study using a case study with qualitative methodology. We used a convenience sampling in which episodic interviews and discussion groups were carried out to 16 preschooler teachers from a north city of Chile. Data was analyzed by using techniques of topic coding and grounded theory. From this analysis, essentialist subjective theories of human nature, infancy and prosociality were found, which support (1) preschooler teacher' subjective theories about how prosociality develops, teaches and learns in young children, in addition to supporting (2) poor pedagogical practices of preschool teachers. Finally, results are discussed in the light of the current literature of prosocial behavior, proposing new areas of topic research and some suggestions to promote education in prosociality.

CÓMO CITAR:

Cicardini, G., Martínez, M., Ramírez, K., Cuadra-Martínez, D., Castro-Carrasco, P. J. y Slomp, N. (2021). Prosocialidad en preescolares: Estudio de teorías subjetivas de profesoras de educación inicial. REICE. Revista Iberoamericana sobre Calidad, Eficacia y Cambio en Educación, 19(3), 83101. https://doi.org/10.15366/reice2021.19.3.006

${ }^{*}$ Contacto: pablocastro@userena.cl

ISSN: 1696-4713

revistas.uam.es/reice 


\section{Introducción}

Vivimos en una sociedad cuyos problemas sociales y ambientales parecen ir en aumento. Más que nunca se requiere educar a la población para aprender a convivir en armonía con el medio social y ambiental. El comportamiento prosocial o de ayuda al otro, constituye un camino prometedor, porque representa la base para que las personas vivan en paz y los grupos sociales se cohesionen y apoyen recíprocamente (Lay y Hoppmann, 2015).

Este comportamiento positivo se relaciona con una menor violencia (Redondo, Rueda y Amado, 2013) y es generador de emociones positivas, resiliencia, calidad de vida y salud psicológica (Arias, 2015; Son y Padilla-Walker, 2019). Incluso en las escuelas se asocia con un mejor desempeño académico de los estudiantes (Inglés, Martínez-González y García-Fernández, 2013), aunque también se ha encontrado una correlación débil y negativa entre prosocialidad y rendimiento académico (Shirin, 2020).

Aunque su delimitación conceptual se encuentra en desarrollo, el comportamiento prosocial se puede entender como las acciones voluntarias cuyo objetivo son beneficiar, apoyar, ayudar o entregar confort a una o más personas (Dunfiel y Kuhlmeier, 2013; Eisenberg, Eggum y Di Giunta, 2010). Desde algunos autores estas acciones podrían ser altruistas (Anl1, 2019; Hepach et al., 2016), mientras que desde otras miradas teóricas constituyen solo una manifestación conductual de ayuda, con independencia del tipo de intención (Auné et al., 2014).

Respecto de cómo se desarrolla la prosocialidad, actualmente se asume que hay múltiples causas (Garaigordobil, 2014). Desde una perspectiva socio cultural, la prosocialidad se desarrolla fundamentalmente mediante procesos de socialización, en donde el infante aprende a ser selectivo en la emisión de conductas de ayuda hacia otros (Tomasello, 2010).

La escuela es uno de los contextos sociales más importantes para el desarrollo, la enseñanza y el aprendizaje de la prosocialidad en los niños y niñas. Para Redondo, Rueda y Amado (2013), la escuela es un espacio con una amplia y variada red de interacciones, que representan una oportunidad para poner a prueba los comportamientos prosociales, la resolución de conflictos, la inclusión y la democracia. Sin embargo, se requiere que en ésta se instalen procesos sistemáticos de desarrollo de la prosocialidad (Garaigordobil, 2014), porque actualmente estas instituciones presentan dificultades para la educación socioemocional, dado que tienden a poner un mayor énfasis en el aprendizaje de contenidos (Arias, 2015).

Para prosperar en la sociedad, los estudiantes deben aprender habilidades sociales y emocionales, entre éstas, desarrollar actitudes positivas hacia la escuela y la comunidad, sobre todo desde la primera infancia, por lo que la educación inicial debe incluir programas cuyo objetivo sea que los estudiantes se desarrollen social y emocionalmente (Lería, Salgado y Sasso, 2018; O'Conner et al., 2017). Sin embargo, esto ha sido poco estudiado (Papadopouloua et al., 2014). De ahí que este trabajo busca contribuir a la comprensión del desarrollo, enseñanza y aprendizaje del comportamiento prosocial en los infantes, desde la perspectiva de los profesores de la educación inicial.

Actualmente se sabe que durante los años escolares los preescolares desarrollan sucesivamente la independencia y la conducta de cooperación. En la medida que se vuelven más verbales se hacen más conscientes de sí mismos y capaces de pensar en el punto de vista de otra persona e interactuar con sus compañeros. Se espera que, al momento de compartir, el niño tenga un adecuado sentido de autoeficacia y la capacidad emocional para empatizar con la otra persona.

Sin embargo, en edades tempranas no todos los infantes manifiestan adecuadamente una conducta prosocial, porque es necesario que interactúen con modelos y experiencias sistemáticas ligadas a recibir ayuda de otros, imitando lo que sus cuidadores han hecho con ellos (Henao, 2014). En general, este tipo de educación se ha abordado de una manera simple y menos planificada, en donde una de las estrategias más utilizadas para enseñar a los niños es la de modelar la conducta prosocial (García, 2010).

Uno de los focos de la educación socioemocional en la infancia es el desarrollo de la consciencia social, que implica enseñar a los infantes a adoptar la perspectiva de los demás y a empatizar, instalando comportamientos de ayuda (O'Conner et al., 2017). En la sala de clases, el educador es quien debe desarrollar en el infante actitudes y habilidades prosociales utilizando para ello estrategias pedagógicas (Campos, 
Chacc y Gálvez, 2006), como la cooperación, juegos didácticos y de resolución de conflictos y materiales para facilitarlo (Acar y Torquati, 2015).

Sin embargo, la evidencia muestra que cuando los profesores abordan fenómenos educativos para los cuales tienen una limitada formación, se orientan en base a teorías subjetivas (TS), la mayor parte del tiempo con una baja conciencia de esto (Cuadra-Martínez et al., 2017). Se requiere que los profesores reciban una formación y una asistencia técnica de calidad para el desarrollo socio emocional del estudiante, que les permita planificar e implementar prácticas pedagógicas sustentadas en saberes procedimentales y teóricos, sobre todo en los profesores de la educación inicial (O’Conner et al., 2017). Es por esto que este trabajo busca comprender el desarrollo, enseñanza y aprendizaje de la prosocialidad, desde el saber subjetivo docente.

\section{Revisión de la literatura}

Aunque hay insuficiente información sobre la forma en que los profesores de la educación inicial inciden en el desarrollo del comportamiento prosocial en los infantes, se sabe que un vínculo positivo profesor- estudiante, favorece de manera directa el desarrollo de este comportamiento en los alumnos(as) (Ferreira et al., 2016).

En relación a lo anterior, los estudios muestran que las creencias de los educadores sobre la infancia, el desarrollo y la enseñanza, inciden en la adopción y uso de diferentes prácticas en el aula (Wang et al., 2008; Wen, Elicker y McMullen, 2011). Para Li y otros (2016), es fundamental estudiar la manera en que los profesores de preescolares comprenden y describen los comportamientos sociales de los infantes, ya que esto posibilita en los educadores la identificación temprana de dificultades en el desarrollo socioemocional de los niños y, desde allí, la instalación de remediales.

Los profesores de educación inicial valoran el desarrollo socioemocional de sus estudiantes, incluyendo creencias de valoración de comportamientos de ayuda y protección entre los escolares (Hollingsworth y Winter, 2013; Sims et al., 2012), siendo considerados estos comportamientos igual o más importantes que la alfabetización o la matemática (Kowalski, Pretti-Frontczak y Johnson, 2001). Otro tipo de creencias encontradas en profesores de preescolares son el valor del juego como estrategia pedagógica y la importancia de considerar en el plan de estudios los intereses, necesidades y experiencia de la vida cotidiana de los niños en edad preescolar, para promover el aprendizaje.

Algunos estudios se han focalizado en las creencias de autoeficacia docente, para la educación socioemocional, encontrando evidencia no concluyente al respecto. Hollingsworth y Winter (2013) advierten que los maestros presentan creencias de una baja autoeficacia para la educación socioemocional, lo que podría constituirse en un obstaculizador de la planificación e instalación de prácticas pedagógicas que promuevan el desarrollo de la prosocialidad en los estudiantes. Sin embargo, también se presenta evidencia de que los profesores de la educación inicial presentan creencias que los orientan a preparar al infante para su progresión a niveles educativos superiores, siendo el desarrollo de la autonomía y de la capacidad para funcionar adecuadamente en un grupo social, uno de los focos de enseñanza (Rimm-Kaufman et al., 2005).

En relación con la prosocialidad, hay evidencia de que en la educación inicial, los profesores de preescolares tienden a experimentar emociones positivas y a aprobar el despliegue de los comportamientos de ayuda de sus estudiantes, mientras que frente a conductas disruptivas, vivencian emociones negativas y despliegan acciones de desaprobación de estos comportamientos (Li et al., 2016).

Por otro lado, esta relativa importancia que los profesores de preescolares otorgan a la educación socioemocional y al comportamiento de ayuda, contrasta con una insuficiente instalación de prácticas sistemáticas para su enseñanza, lo que los profesores explican apelando a factores que principalmente escapan de su control, como las políticas educativas y las condiciones socio familiares de sus estudiantes (Papadopouloua et al., 2014). Otra posible explicación es que los profesores se disponen a atender, apoyar y educar socioemocionalmente más a aquellos estudiantes que presentan comportamientos disruptivos al interior del aula, mientras que aquellos que despliegan prácticas prosociales son menos atendidos (Ar- 
beau y Coplan, 2007), lo que podría estar asociado al hecho de que este último grupo de infantes favorece ambientes de aula que facilitan la tarea educativa del docente.

Lo anterior, también podría deberse a que los profesores en la educación de preescolares, tienden a tomar decisiones educativas sobre el desarrollo socioemocional de los infantes, guiados en gran parte por sus creencias y no tanto en base al conocimiento formal. Esta situación la explican McLaughlin, Aspden y Clarke (2017) en base a los limitados conocimientos y estrategias de enseñanza que han adquirido para abordar de manera sistemática y planificada la educación socioemocional. Además, la tarea de educar socioemocionalmente a preescolares es compleja y, según Kirk y Jay (2018), es necesario que el profesor logre integrar aspectos propios del entorno de enseñanza, del juego y de las relaciones interpersonales para favorecer sistemáticamente el desarrollo socioemocional del infante. Cuando uno de estos elementos no es integrado adecuadamente, se limita la eficacia de los apoyos sociales y emocionales para las y los niñas(os).

Los hallazgos encontrados en la literatura sobre el tema, advierten de la relevancia de indagar más sobre las creencias a la base que regulan las decisiones y prácticas educativas prosociales, de los docentes de la educación inicial. Para comprender el desarrollo, enseñanza y aprendizaje de la prosocialidad en los preescolares, desde el saber subjetivo de los educadores, este trabajo aborda un tipo particular de creencia, las teorías subjetivas, cogniciones acerca de sí mismo y del mundo, que tienen una estructura argumentativa hipotética, por lo menos parcialmente explícita y que orientan el comportamiento (Catalán, 2016; Flick, 2014).

Las TS tienen un rol importante en la forma en que los profesores representan fenómenos educativos y orientan sus prácticas pedagógicas (Cuadra-Martínez et al., 2017). En la misma línea, Kelchtermans (2009) plantea la existencia de una teoría educativa subjetiva.

Los docentes de carreras desarrollan un marco interpretativo personal: un conjunto de cogniciones, de representaciones mentales que opera como un lente a través del cual los docentes miran su trabajo, le dan significado y actúan en él. Por lo tanto, este marco guía sus interpretaciones y acciones en situaciones particulares (contexto), pero al mismo tiempo también se modifica y resulta de estas interacciones significativas (toma de sentido) con ese contexto. (pp. 260-261)

En función de lo anteriormente planteado, como problema de estudio se busca responder ¿cuáles son las TS sobre el desarrollo, la enseñanza y el aprendizaje del comportamiento prosocial en preescolares, que presentan los(as) profesores(as) de la educación inicial? Así, el objetivo general es describir e interpretar estas TS y a nivel específico: (a) describir el significado subjetivo del comportamiento prosocial de estos participantes; (b) determinar las explicaciones que presentan acerca del desarrollo, enseñanza y aprendizaje del comportamiento prosocial; (c) describir, desde sus TS, las prácticas pedagógicas que implementan para promover el desarrollo de la prosocialidad en sus estudiantes y (d) proponer un modelo comprensivo del desarrollo, enseñanza y aprendizaje de la prosocialidad en la educación preescolar.

\section{Método}

\section{Enfoque metodológico}

Corresponde a un estudio descriptivo interpretativo, basado en el método cualitativo. En este tipo de estudios es fundamental abordar la subjetividad para comprender de forma más profunda, natural y holista el problema de estudio (Taylor y Bogdan, 2000). Se utilizó un diseño de estudio de casos, a fin de caracterizar profundamente el problema de estudio, desde la particularidad y complejidad de un caso singular (Stake, 1995).

\section{Participantes}

Los participantes fueron 16 educadoras de párvulos (título universitario que reciben en Chile los docentes de educación preescolar) que se seleccionaron de acuerdo a un muestreo intencional y por conveniencia (Flick, 2014), bajo el criterio de representatividad de casos, considerando distintos años de servicio, dis- 
tintos niveles educativos de atención y distintos rangos de edad: ocho pertenecen a dos jardines infantiles públicos y ocho a dos jardines infantiles particulares de una región del norte de Chile. La muestra presentó en promedio 32 años de edad y 3 años de ejercicio profesional. Como criterios de inclusión se consideró: (a) contar con el título profesional de Educador(a) de Párvulos, (b) estar ejerciendo actualmente su profesión en el aula, y (c) tener como mínimo un año de servicio profesional.

\section{Trabajo de campo e instrumentos de obtención de información}

Para la recolección de la información, se contactó a las educadoras, a quienes se les dio a conocer el trabajo investigativo y los criterios éticos, lo que se concretó con la firma de un consentimiento informado. Se aplicaron dos instrumentos de recolección de información.

Entrevista episódica. El elemento central de esta forma de entrevista es la invitación periódica a presentar narraciones de situaciones y experiencias a fin de reconstruir TS desde éstas (Flick, 2014). Para su implementación, se elaboró un guion temático que consideró los siguientes tópicos: a) significado de prosocialidad, b) desarrollo de la prosocialidad en preescolares, c) enseñanza y aprendizaje de la pro-socialidad en preescolares, y d) didácticas y estrategias de enseñanza de la prosocialidad en preescolares. Se aplicaron 8 entrevistas episódicas en instalaciones del jardín infantil, tuvieron una duración aproximada de 1 hora y se grabaron en audio (Cuadro 1).

Grupo de discusión. Este instrumento también se basó en la técnica de la entrevista episódica, dado que se ha visto que la narración de experiencias en contexto grupal también es un fuerte elicitador de TS (Cuadra-Martínez, 2016). Además, incluyó el mismo guion temático de la entrevista episódica, con al-gunas variaciones para contrastar y profundizar en comprensión los hallazgos obtenidos a partir de las entrevistas. Se aplicaron 2 grupos de discusión, con una duración aproximada de 1 hora cada uno y los datos se grabaron en audio.

La opción metodológica de utilizar dos instrumentos apuntó a propiciar la elaboración de una teoría comprensiva del fenómeno estudiado, en la medida que los datos se fueron recabando de manera sucesi-va y simultáneamente analizando, lo que orientó el proceso de recolección de datos siguiente. Esto tam-bién permitió satisfacer el criterio de credibilidad, al triangular no solo observadores, sino que además instrumentos.

Cuadro 1

Aplicación de los instrumentos

\begin{tabular}{lcc}
\hline & Municipal & Particular \\
\hline Entrevista episódica & 4 & 4 \\
Grupo de discusión & 4 & 4 \\
\hline
\end{tabular}

Nota. Elaboración propia.

\section{Análisis de datos}

El análisis de datos se basó en una combinación de la técnica de análisis de la teoría fundamentada (Strauss y Corbin, 2002) y el procedimiento de descripciones breves de casos de la codificación temática (Flick, 2014):

Análisis intracaso. Para cada entrevista y grupo de discusión se elaboró un lema representativo del texto, una breve descripción del caso y una síntesis de los temas principales del texto. En este proceso se identificaron TS (codificación abierta), lo que implicó reconstruir una estructura de hipótesis o teoría, identificando un antecedente y un consecuente, en la mayoría de los casos utilizando la forma si... entonces; porque... (Catalán, 2016). De esta manera se identificaron frases o párrafos textuales que dieron origen a códigos expresados por los investigadores.

Análisis intercaso. Se compararon todos los casos analizados en la primera etapa, para construir una estructura temática común. Este proceso se realizó codificando para depurar y diferenciar las categorías derivadas de los códigos construidos (Flick, 2014) y los análisis intracaso, lo que permitió determinar las dimensiones y contenidos de las categorías más relevantes para el problema de estudio (Flick, 2014). 
Codificación axial. Posteriormente, la codificación se orientó a la identificación de TS supra ordenadas que incluyeran a subcategorías relacionadas, además de establecer relaciones entre ellas, buscando un mayor nivel de abstracción e interpretación en el análisis.

Codificación selectiva. Finalmente, los investigadores identificaron una categoría central, en torno a la cual relacionaron las categorías reconstruidas mediante la codificación axial, lo que permitió elaborar una teoría comprensiva del fenómeno estudiado.

En el apartado de resultados se presenta una tabla que sintetiza los análisis intracaso. Posteriormente, cada tema o categoría se presenta como resultado de la codificación abierta, seguida por su correspondiente codificación axial, que se expone en base a un breve texto y un mapa conceptual. Finalmente, se presenta la codificación selectiva siguiendo la sugerencia de Flick (2014), de presentar un breve texto y una figura de relaciones de conceptos.

\section{Resultados}

\subsection{Análisis intracaso}

A continuación, se presenta una síntesis del análisis intracaso, agrupando por tipo de institución educativa. Por razones de espacio, se describe cada caso, el lema correspondiente y la TS sobre el comportamiento prosocial que más lo representa (Cuadro 2).

Cuadro 2

Análisis intracaso jardines infantiles particulares y públicos

\begin{tabular}{ccc}
\hline Caso & Lema & TS \\
\hline
\end{tabular}

Jardín infantil particular

1: N., educadora de párvulos de 26 años de edad. Tiene dos años de experiencia laboral en el jardín particular A.

La enseñanza prosocial preescolar es la base del desarrollo del adulto

2: M., educadora de párvulos de 27 años de edad. Tiene tres años de experiencia laboral en el jardín particular A.

3: G., educadora de párvulos, de 32 años de edad. Tiene tres años de experiencia laboral en el jardín particular A.

4: M., educadora de párvulos, de 35 años de edad. Tiene tres años de experiencia laboral en el jardín particular A.

5: Grupo de discusión Jardín particular A., participaron 4 educadoras de párvulo de diversas edades.
La familia es la base del desarrollo prosocial

Incentivos para el desarrollo del comportamiento prosocial

La cotidianeidad para la enseñanza y aprendizaje de la prosocialidad

La educación parvularia como complemento de la educación parental prosocial

El comportamiento prosocial se expresa sin esperar retribuciones y sin hacer distinciones.

Consiste en ayudar desinteresadamente y se desarrolla a través de una interacción intencionada, en donde la familia es la base y un ejemplo a imitar. Esto fomentaría el desarrollo de este comportamiento y su prolongación hasta la adultez, asociándose además con el desarrollo personal.

El comportamiento prosocial parte de aquella necesidad humana de compartir con el otro, entonces se deben crear instancias en los niños para esto.

Este comportamiento se desarrolla principalmente en el núcleo de la familia, donde los niños aprenden a través de la imitación, instancias de estimulación, exploración y aprendizaje de este comportamiento.

Este comportamiento se desarrolla evolutivamente y en base a incentivos proporcionados en el núcleo familiar y la escuela.

Entonces, cuando la familia y la escuela generan un ambiente motivador, sano y ejemplar en valores, el niño(a) progresivamente se torna prosocial.

“... es como un comportamiento mutuo, de compartir con otro diariamente, ayudándose mutuamente, donde las educadoras serán aquella figura de guía constantemente, incentivando y creando un hábito donde se espera desarrollar el compartir, siempre desde lo cotidiano”.

El comportamiento prosocial se desarrolla a través de un equilibrio entre la educación impartida en el jardín infantil y la impartida por la familia. Se requiere que, a través de la imitación, el adecuado uso del lenguaje y el juego, el niño pueda desarrollar su autonomía, seguridad, independencia, autoestima y autoeficacia, llegando así a convertirse en una persona prosocial. 


\section{Jardín infantil público}

6: K., educadora de párvulos, de 26 años de edad. Tiene cuatro años de experiencia laboral en el jardín público $\mathrm{C}$. párvulos, de 42 años de edad. Tiene tres años de experiencia laboral en el jardín público C.

8: V., educadora de párvulos de 33 años de edad. Tiene dos años de experiencia laboral en el jardín público $\mathrm{C}$.

9: D., educadora de párvulos, de 27 años de edad. Tiene dos años de experiencia laboral en el jardín público C.

10: Grupo de discusión Jardín público U., participaron 4 educadoras de párvulo de edades diversas.
Prosocialidad como comportamiento innato en la infancia, pero que requiere educación para su desarrollo

\section{Prosocialidad} para la bondad humana y el crecimiento personal

Prosocialidad, familia y escuela: rendirse jamás

Empatía y colaboración para la prosocialidad: los infantes pueden ser prosociales

Reforzando la prosocialidad mediante normas, límites, juegos e imitación: alianza familia escuela
"Con prosocial me imagino como ir abanderado con las cosas sociales, es algo innato en los niños, ellos tienen esta cualidad, pero como adultos en vez de guiarla hacia el lado bueno, la guiamos al lado malo. Enseñar a ser prosocial es enseñar a ser persona, es tener valores...”.

\begin{abstract}
"Un comportamiento en donde las personas mediante sus valores se relacionan con sus pares de buena forma. Es base para hacer crecer a buenas personas, crear buenos sujetos para la sociedad, junto con sus valores se puede generar un cambio grande y favorable”.
\end{abstract}

\begin{abstract}
"Es un comportamiento en donde los niños aprenden por la familia y nosotras vamos guiando día a día”.

"No hay que tirar la esponja, solo hay que ir reforzando y recordar que se va reforzando esto día a día. Una no se debe rendir nunca".
\end{abstract}

“...aquel comportamiento que se inculca, se enseña o potencia en el jardín, que se trabaja en conjunto con los papás, y es un aprendizaje que los niños deben desarrollar y que tienen las capacidades, partiendo de la base del conectarse con uno mismo, para ponerse en el lugar del otro, desarrollando la empatía y la colaboración"

P2: "Es transversal, tiene normas y límites que se refuerzan, hábitos, siendo un indicador de convivencia, se va generando mediante juegos e imitación. P3: "La base es la familia, porque es donde se desarrolla el niño, luego de eso viene la parte de la educación, con sus agentes, entonces no hay una cosa sin la otra”.

Nota. Elaboración propia.

El análisis comparativo de cada uno de los casos permitió elaborar un eje temático común a cada uno de ellos mediante el análisis intercaso y la codificación abierta, lo que se describe a continuación.

\subsection{Significado subjetivo del comportamiento prosocial}

El significado que las educadoras le otorgan a la prosocialidad se encuentra relacionado con tres TS: la primera es una TS esencialista sobre la naturaleza humana y el niño(a); la segunda, una TS esencialista sobre el comportamiento prosocial y la tercera, explica el desarrollo de este comportamiento.

Se observa una TS esencialista sobre la naturaleza humana y la infancia, en donde se caracteriza al ser humano por su capacidad para reflexionar, razonar y pensar, atributos que son extensivos a la infancia, pero con diferencias importantes en los niños(as) relacionadas con la prosocialidad.

Según las participantes del estudio, los adultos se caracterizan por un mayor sentido de la responsabilidad, asociando esto a una inflexibilidad en su forma de pensar y autoritarismo en la relación con los niños(as), lo que conllevaría a una menor posibilidad de cambio y disposición a ayudar a otros. Los niños(as), en cambio, son definidos como seres esencialmente inocentes y bondadosos, que tienen un potencial enorme para aprender y cambiar, por lo que presentan mayores comportamientos de apoyo y colaboración que los adultos. 
Ser niño es disfrutar, jugar, aprender jugando, aprender compartiendo, ser una persona con opinión, porque eso ha costado un poco porque el adulto tiene esta posición adultista que generalmente no le permite al niño expresarse ni tener opinión sobre algunas cosas. (Participante 4, caso 9)

También se presenta en las educadoras una TS esencialista de la prosocialidad, que explica que el comportamiento prosocial es parte de la especie humana, porque es necesario para la sobrevivencia. Se encuentra más presente en la infancia, dado que el niño(a) tiene una capacidad intrínseca para identificar la necesidad de ayuda y reconocer las emociones de los demás.

El ser humano está condicionado a hacer esas cosas, bueno primero fue por un tema de sobrevivencia, pero el ser humano necesita compartir para tener experiencias, para aprender, para poder sobrevivir, entonces tenemos que crearles a los niños instancias para que puedan compartir. (Caso 2)

La TS sobre el desarrollo del comportamiento prosocial, explica que este comportamiento, si bien es parte de la persona, requiere de tres condiciones para que pueda manifestarse en los niños(as). En primer lugar, es necesario que el infante logre una madurez cognitiva suficiente para poder identificar y comprender este comportamiento, lo que se lograría con tiempo y una estimulación adecuada. Así lo expresa el caso 1:

Puede que lo traigan, pero también puede irse desarrollando con el tiempo, con lo que van viendo a su alrededor, lo que ven de los adultos, que es lo que ellos imitan, pueden traerlo, pero se va desarrollando con el tiempo también. (Caso 1)

En segundo lugar, se requiere de una educación formal que valore y sistematice la enseñanza de la prosocialidad. Las educadoras se atribuyen la responsabilidad de incentivar, potenciar y mantener las conductas prosociales de los infantes, utilizando normas y límites, formando hábitos e incorporando a la familia en este proceso. La participante 4 del caso 10 señala:

... uno le va enseñando, o sea es algo que nosotros le vamos inculcando, la familia, porque este trabajo va de la mano, hay niños que tienen esa habilidad de ayudar al compañero, pero en sí el resto lo aprende. (Participante 4, caso 10)

En tercer lugar, es necesaria la educación parental, en donde el grupo familiar modele, oriente y refuerce los comportamientos de ayuda a otros. La familia es quien tiene un rol fundamental en el desarrollo de la conducta de ayuda, siendo el jardín infantil un contexto que apoya este aprendizaje, como se ejemplifica en el caso 6:

... la familia (...) [porque] piensa que el jardín está encargado de todo, [entonces] esto es algo netamente de casa, desde la casa deberían venir con estas conductas adquiridas.

(Caso 6)

\subsubsection{Codificación axial del significado subjetivo de la prosocialidad}

El ser humano es esencialmente colaborador, requiere de este comportamiento para avanzar en su desarrollo y el niño es un ser definido como ontológicamente bondadoso y solidario. Sin embargo, para que se manifieste el comportamiento de ayuda a otros, se requiere gestar ciertas condiciones en la familia, escuela y otros contextos de socialización, a fin de que el niño "madure" cognitivamente, respetando sus tiempos o ritmos de aprendizaje e instalando una enseñanza sistemática del comportamiento de ayuda. Cuando no se logran instalar estas condiciones, entonces en el niño(a) este comportamiento podría manifestarse menos, conllevando al desarrollo de adultos inflexibles, autoritarios y menos solidarios (Figura 1). 
Figura 1

Codificación axial del significado subjetivo del comportamiento prosocial

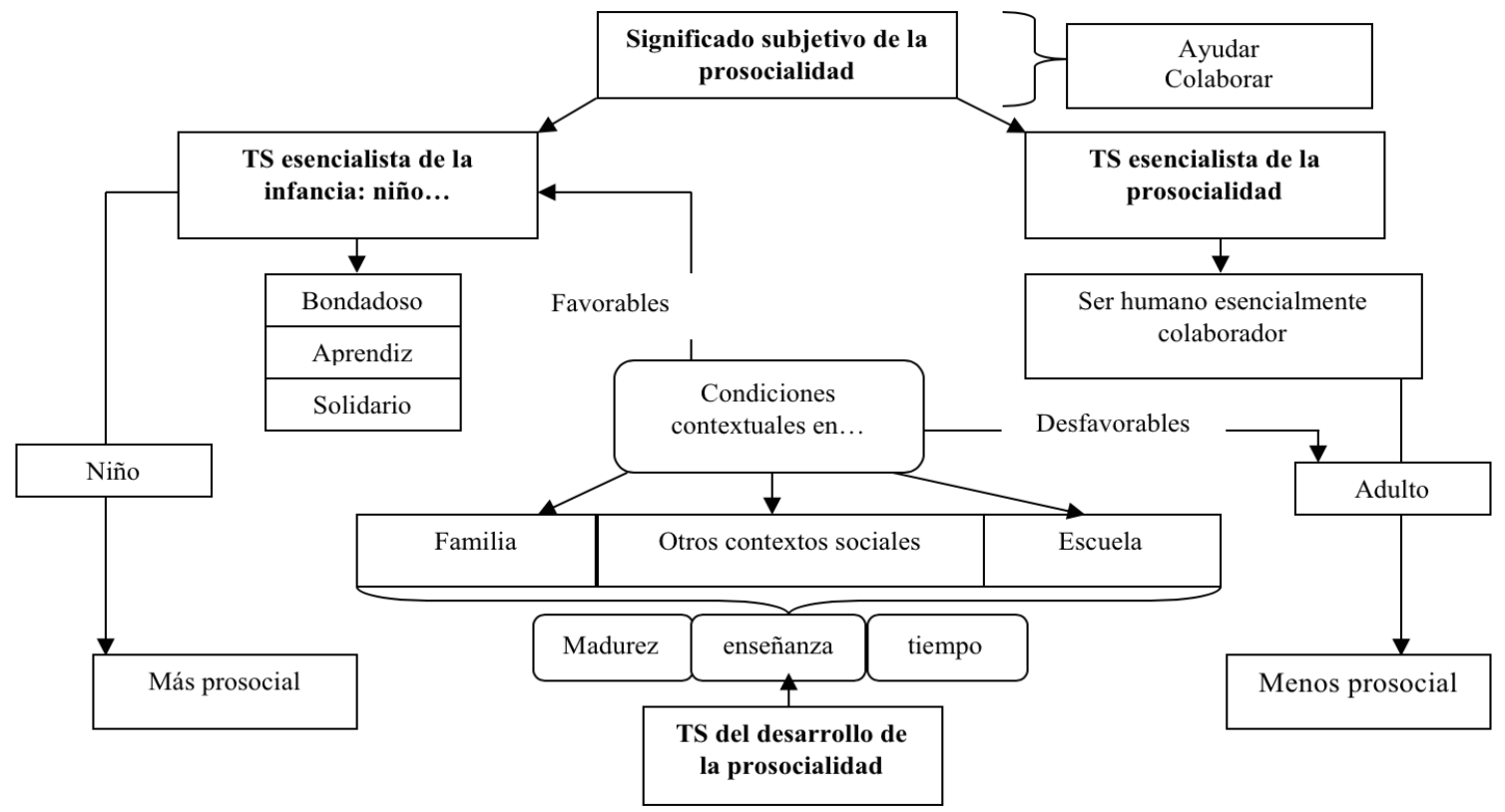

Nota. Elaboración propia.

\subsection{Teorías subjetivas sobre la enseñanza y aprendizaje del comportamiento pro- social}

Cuatro TS se presentan en las educadoras de párvulo, acerca de la enseñanza y aprendizaje del comportamiento prosocial en los preescolares. La primera TS posee un alto contenido emocional y orienta la acción hacia la enseñanza de la prosocialidad en las educadoras. Las educadoras le atribuyen una alta importancia al comportamiento prosocial, asociándolo con el desarrollo de niños(as) saludables, capaces de trabajar en equipo y de contribuir a la sociedad. Desde esto, en algunos modelos educativos de los jardines infantiles la solidaridad o ayuda a otros se presenta como una meta a lograr. Así lo expresan algunas educadoras:

Mucho, mucho, de hecho, lo tenemos declarado en nuestro proyecto educativo, de que los niños compartan... (Participante 5, caso 10)

Esto es importante porque después esto ayuda cuando ellos sean adultos, a desenvolverse con facilidad en la sociedad, para tener buenos ambientes laborales, personales. (Caso 1)

La segunda TS se puede esbozar de la siguiente manera: para que el infante pueda aprender el comportamiento prosocial, se requiere de una enseñanza lo más natural posible, porque es en la cotidianeidad en donde los niños(as) ponen a prueba sus formas de interactuar. La enseñanza y aprendizaje del comportamiento prosocial en los preescolares es entendida como un proceso intencionado, que varía en el grado de sistematización, siendo más adecuado instalarla de la forma más natural posible, donde los tipos de ayuda se manifiesten y pongan a prueba en contextos reales de actuación. Desde esta perspectiva, las educadoras consideran que actualmente las formas de enseñar se alejan cada vez más de la formación socioemocional y esto se agrava en la medida que los estudiantes avanzan en niveles educativos, lo que este grupo denomina como escolarización de la educación. Así lo expresan las educadoras del caso 10:

[En enseñanza básica] No hacen seguimientos como para ver si cambia o no con lo que enseñan los colegas, pero se sabe que es distinto. Porque [en el jardín infantil] todo se hace en base al juego, para él es un cambio, porque es otro ambiente, otra situación. (Participante 1 , caso 10) 
Aquí entregamos a los niños con sus mamás y los esperamos ahí en el colegio los entregan en la puerta y eso es un cambio radical y fuerte para el niño. Somos más maternalistas que en el colegio, por eso los papás notan el cambio. (Participante 3, caso 10)

En la tercera TS, las educadoras explican que la enseñanza y aprendizaje del comportamiento prosocial es un proceso complejo que "significa enseñar a ser persona” (caso 6). Así, las educadoras señalan que es necesario abordar integralmente al infante, considerando el aprendizaje de valores, de la empatía y de conocimientos procedimentales relacionados con el comportamiento de ayuda.

A través de juegos, lo que haces diariamente, a los más pequeños se les enseña desde el hablar, como decirle no le pegues al compañero... es fundamental enseñarle en esta etapa, sobre todo por la adquisición de valores es importante para que los niños crezcan bien. (Caso 3)

Nuestro sello pedagógico es educar en emociones en sintonía con el ambiente, entonces nosotros trabajamos mucho de que, si se cae un niño, por ejemplo, no se debe reír... (Caso 6)

La cuarta TS identifica factores que son facilitadores y obstaculizadores de la enseñanza y aprendizaje de la prosocialidad. Se señala como facilitadores, (a) la figura de la educadora como tal, dado que tiene un rol educativo y mediador de importancia en la enseñanza y aprendizaje del niño, (b) un ambiente de enseñanza y aprendizaje motivador y adecuado a las necesidades del infante, y (c) un adecuado ejercicio de la crianza del infante.

Como facilitadores, creo que sería un ambiente propicio para que los niños estén y alguien que los incentive siempre y les esté recordando que deben ser buenos compañeros, además de una buena decoración de la sala de clases, es decir es necesario un ambiente completamente motivador. (Caso 3)

Como obstaculizadores, se señalan dos tipos: (a) un núcleo familiar de límites rígidos, con una limitada relación con el medio y una constitución familiar de hijo único, que no permite que el niño socialice con otras personas, obstaculizando el aprendizaje de la prosocialidad, condición familiar que para las educadoras va en aumento; (b) mientras que en el contexto de educación formal preescolar, los obstaculizadores apuntan a la cada vez mayor presión desde las políticas educativas, por enseñar contenidos, en desmedro de la educación socioemocional. Para las educadoras, ambos obstaculizadores estarían más presentes en los jardines particulares, respecto de los públicos. Así lo expresan los participantes:

... pero he visto diferencias en jardines municipales y particulares y eso marca mucho, vi mucha diferencia incluyendo a los padres que marcan bastante la base de los niños a la hora de compartir. (Caso 7)

Los jardines están súper escolarizados, [entonces] el niño se sienta con un libro y trabaja y muchas veces no compartimos lo que hace con los demás, el lápiz, cada uno trae su estuche. (Caso 2, jardín infantil particular pagado)

La enseñanza y aprendizaje de la prosocialidad en los preescolares, se regula en algún nivel en base a 4 TS que la sustentan. La mayoría de estas TS son iniciadoras y mantenedoras de la acción de enseñanza de la prosocialidad, lo que significa que orientan sus prácticas pedagógicas al desarrollo de este comportamiento en el infante. No obstante, es posible que cuando la enseñanza y aprendizaje de la prosocialidad no logra avances significativos en los párvulos, las educadoras recurran a justificaciones principalmente externas, que escapan de su control, lo que podría inhibir sus esfuerzos por remediar los resultados obtenidos (Figura 2). 
Figura 2

Codificación axial: TS sobre la enseñanza y aprendizaje de la prosocialidad

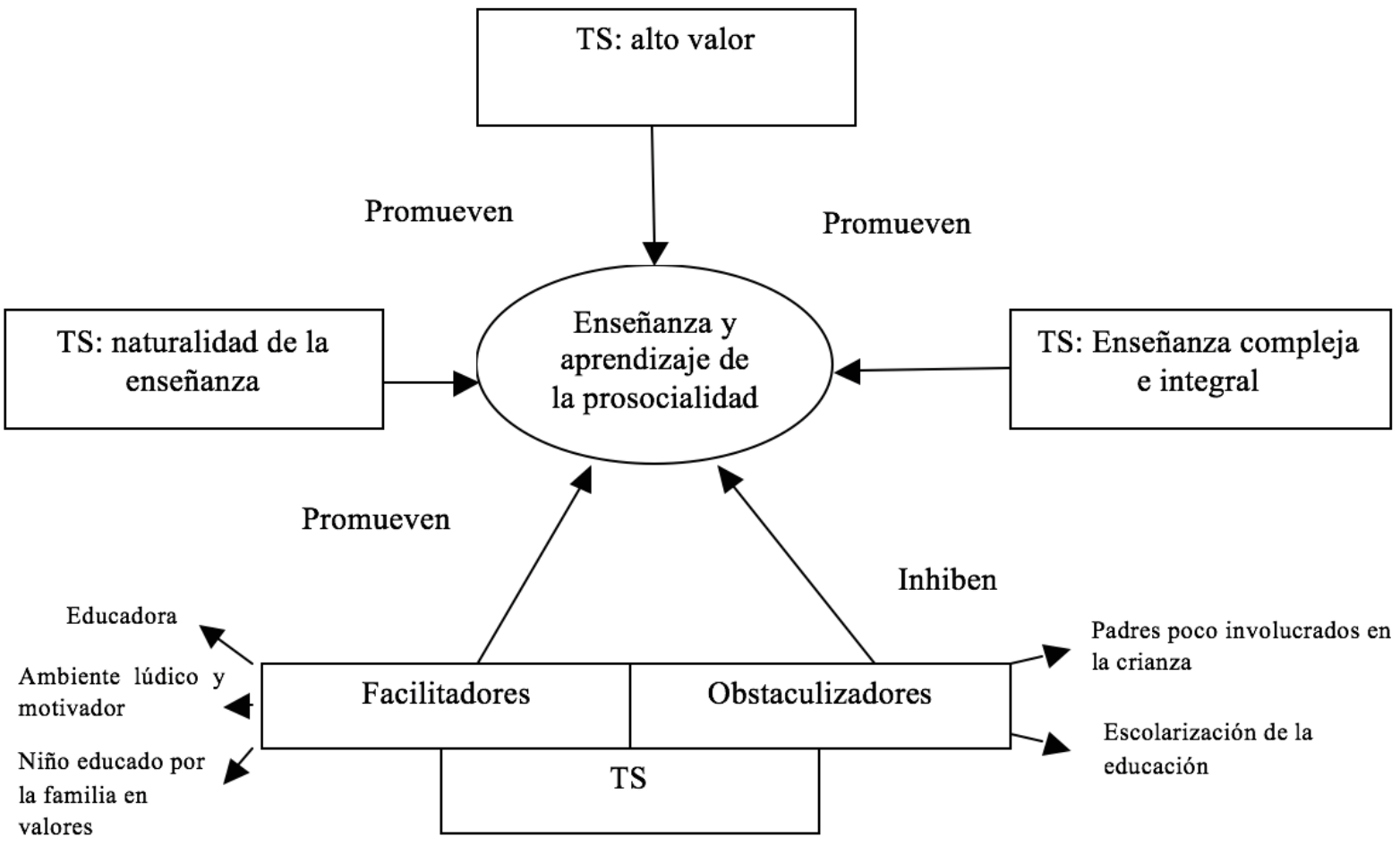

Nota. Elaboración propia.

\subsection{Prácticas pedagógicas para la enseñanza de la prosocialidad}

Las prácticas pedagógicas de las educadoras oscilan entre situaciones de enseñanza y aprendizaje de la prosocialidad sistematizadas y prácticas espontáneas. Las primeras, son parte de la planificación de sus clases, con base en el currículum de educación parvularia orientadas al desarrollo de valores; mientras que las segundas, emergen en contexto de recreo y de aula, en situaciones de interacción alumno-alumno y alumno-educadora. Así lo expresa el caso 3:

... es algo que surge espontáneamente, en el currículum no hay algo planificado, pero sí hay actividades sobre temas valóricos. (Caso 3)

Las estrategias de enseñanza y aprendizaje de la prosocialidad incluyen una mezcla entre lo lúdico, cotidiano, anecdótico y lo normativo, la puesta de límites y de reglas, combinación que las educadoras hacen con una menor planificación.

En la convivencia diaria, se encuentran actividades ligadas al saludo y despedida, roles de colaboración en el aula y la promoción de compartir sus pertenencias. El uso de canciones busca fomentar el orden, la disciplina y obediencia, además de la inculcación de hábitos de sana convivencia como saludar, despedirse, solicitar perdón, permiso y agradecer. También se fomenta el aseo personal y del jardín infantil, con base en un pensamiento ecológico.

\section{... botar un papel al basurero ya estás ayudando al planeta, o si tu compañero se cayó se da una ayuda de compañerismo, es algo que se ve todo el día, entonces en todo momento ellos se están ayudando, es algo que todo el día se practica. (Caso 5)}

Las prácticas pedagógicas para la enseñanza de la prosocialidad oscilan entre un polo de prácticas asistemáticas y otro polo sistemático y planificado. En una misma línea, se incluyen herramientas lúdicas, juegos y canciones y, por otro lado, límites, normas y reglas para educar en prosocialidad. Esta conjugación entre una enseñanza más formal y prácticas de enseñanza más cercana a la cotidianeidad o a la con- 
vivencia diaria, se entremezclan como recursos que las educadoras utilizan para educar a los niños(as) en el comportamiento prosocial (Figura 3).

Figura 3

Codificación axial de las prácticas pedagógicas de la enseñanza de la prosocialidad

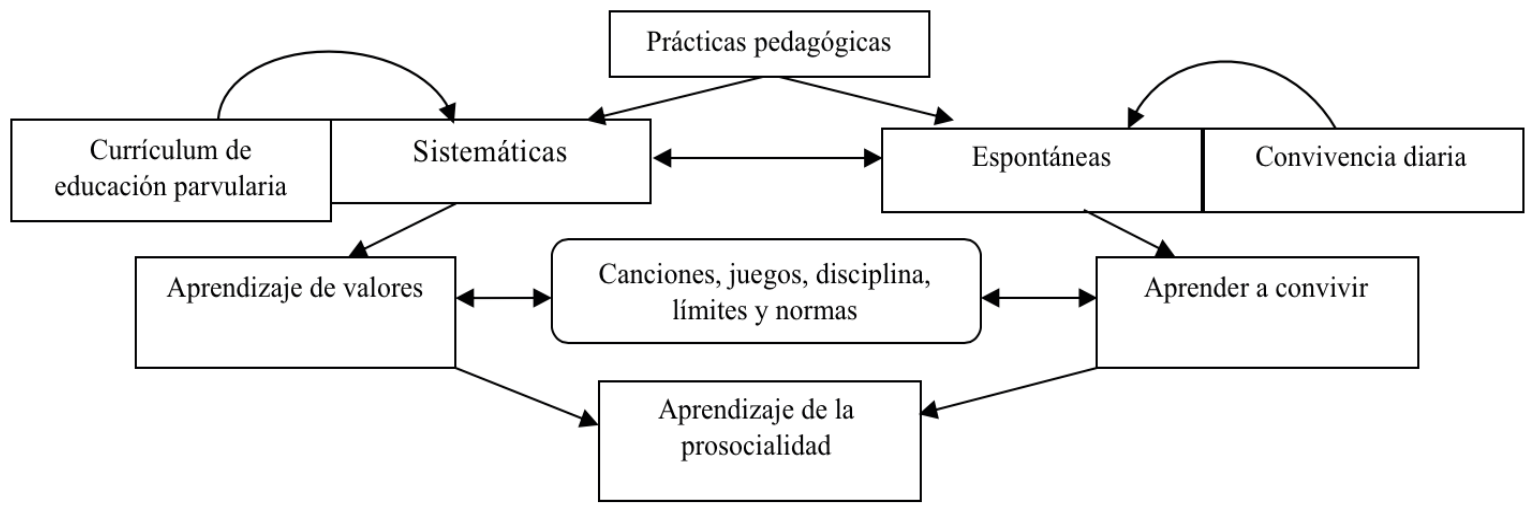

Nota. Elaboración propia.

\subsection{Codificación selectiva: Modelo comprensivo del desarrollo, enseñanza y aprendizaje de la prosocialidad, desde las TS de educadoras de párvulos}

Se presentan tres niveles epistemológicos de TS. El primer nivel implica asunciones ontológicas de la bondad humana, la infancia y la sociedad, que se organizan en base a TS supraordenadas y determinan las TS de la enseñanza y aprendizaje de la prosocialidad. Para que el o la educador(a) se disponga a la enseñanza y aprendizaje de la prosocialidad, es necesario que estas TS supra ordenadas definan al ser humano como esencialmente bondadoso y se considere a la prosocialidad como algo trascendente o importante. También es necesario creer que este aprendizaje es posible, porque el considerar la existencia de un contexto social adverso en la promoción de la prosocialidad, podría constituirse en un inhibidor de la enseñanza y aprendizaje de este comportamiento. En el segundo nivel se encuentran TS acerca de cómo se enseña y cómo aprenden los infantes a ser prosociales, destacando la naturalización de la enseñanza y la consideración de lo complejo que es enseñar a ser prosocial, lo que orienta a pensar en una enseñanza integral para el aprendizaje de este comportamiento. Este nivel, finalmente subordina saberes procedimentales u orienta la práctica profesional, presentándose una integración entre la enseñanza sistemática y formal y la espontánea o cotidiana, lo que impulsa a la instalación de prácticas pedagógicas para el aprendizaje de la prosocialidad o a la generación de espacios para que se practique en la convivencia diaria (Figura 4). 
Figura 4

Codificación selectiva: Modelo comprensivo de la enseñanza y aprendizaje de la prosocialidad

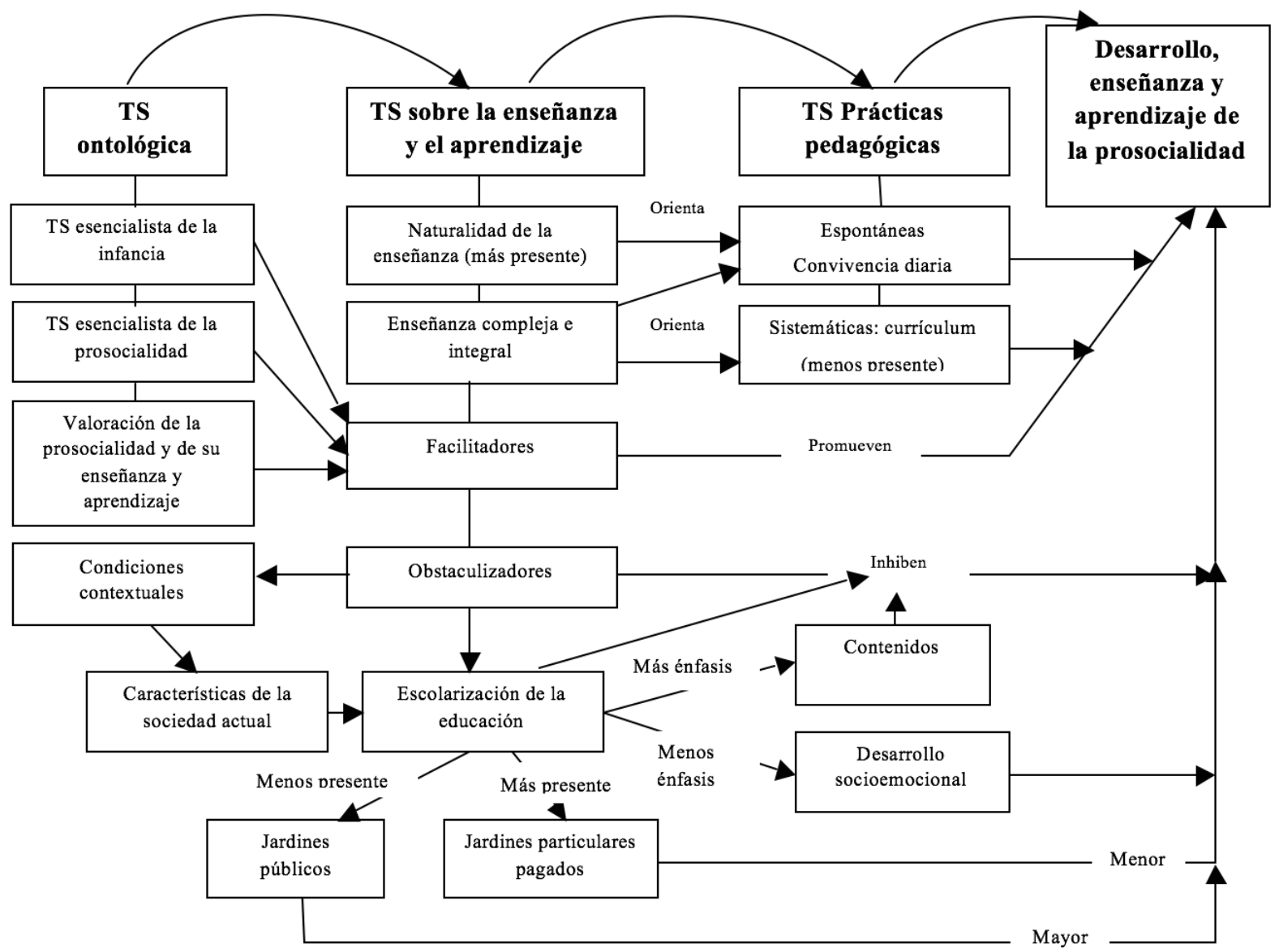

Nota. Elaboración propia.

\section{Discusión y conclusiones}

Este trabajo buscó describir e interpretar las TS de las educadoras de párvulos acerca del desarrollo, enseñanza y aprendizaje de la prosocialidad. Se encontraron TS que las educadoras utilizan para abordar la educación socioemocional y desarrollar la prosocialidad de sus estudiantes. Hallazgo similar se ha evidenciado en otros estudios que abordan la subjetividad del profesor (Cuadra-Martínez et al., 2017; Hollingsworth y Winter, 2013; Papadopouloua, et al., 2014; Sims, et al., 2012; Wang, et al., 2008; Wen, Elicker y McMullen, 2011), lo que advierte de la importancia que este saber subjetivo tiene en la formación y ejercicio profesional y de la necesidad de seguir comprendiendo su incidencia en el desempeño profesional (Cuadra-Martínez, Castro y Juliá, 2018).

Con respecto al significado subjetivo (Flick, 2014) que estas educadoras le otorgan a la prosocialidad, destacan TS esencialistas u ontológicas de la naturaleza humana, del infante y la prosocialidad, en donde se define al ser humano como esencialmente bondadoso y al infante con una mayor disposición a la prosocialidad, destacando el valor que tiene este comportamiento para el progreso social. Parece ser que este tipo de saber subjetivo se organiza en un nivel cognitivo más profundo y regula de manera importante el abordaje de la prosocialidad en un contexto educativo. Asumir al ser humano como esencialmente bondadoso y otorgarle valor al comportamiento prosocial es una condición necesaria para disponerse a desarrollar en otras personas el comportamiento prosocial (Chinea, 2015; Roche, 1997), y en contexto educativo, estas asunciones esencialistas podrían ser claves para que el educador se oriente a promover la prosocialidad en los estudiantes. Lo anterior, dado que permite dar un marco común valórico que facilita la puesta en común de una comunidad educativa, por ejemplo, 
en la relación familia- escuela. Estudios anteriores han mostrado cómo la visión compartida o no, respecto a aspectos transversales de la formación de los párvulos es un tema relevante para educadoras de párvulos (Castro et al., 2019).

En cuanto al desarrollo de la prosocialidad, las educadoras asumen un grado de innatismo de este comportamiento. De acuerdo a Correa (2017) teorías de la personalidad que utilizan el constructo de rasgos, afirman que hay individuos con disposiciones innatas o adquiridas que contribuyen a la conducta de ayuda. Otros modelos de tipo humanista, se fundamentan en posiciones filosóficas previas que han sostenido la existencia en el ser humano de un sentimiento natural y solidario que se manifiesta en la conducta altruista. Sin embargo, desde estos hallazgos sólo bajo ciertas condiciones la prosocialidad se puede desarrollar en el infante. La familia y la escuela son contextos sociales que deben generar experiencias de aprendizaje prosociales, adecuadas a las necesidades y desarrollo evolutivo del infante. Es fundamental la interacción social para que se desarrolle la prosocialidad, especialmente al interior del grupo familiar (Sánchez-Queija, Oliva y Parra, 2006), pero se requiere que estas experiencias de aprendizaje en contextos educativos formales e informales logren algún grado de sistematización, frente a lo cual es preciso fortalecer la formación docente para la educación socioemocional (O'Conner et al., 2017) y educar a la familia para que logre implementar adecuadamente el desarrollo emocional del infante y formar hijos e hijas más prosociales.

Con respecto a la enseñanza y aprendizaje de la prosocialidad, las educadoras relacionan estas prácticas con la formación de niños más competentes para interactuar, con una mejor salud psicológica, y en la adultez, con una adecuada contribución social. Actualmente, la literatura científica ha sido consistente al asociar este comportamiento con una mejor convivencia social (Lay y Hoppmann, 2015), la disminución de la violencia (Redondo, Rueda y Amado, 2013), la salud mental (Arias, 2015) y el éxito académico (Inglés, Martínez-González y García-Fernández, 2013). También se ha presentado evidencia de que los profesores tienden a valorar la educación en prosocialidad (Hollingsworth y Winter, 2013; Kowalski, Pretti-Frontczak y Johnson, 2001; Sims et al., 2012).

No obstante, lo anterior, el problema parece ser la complejidad de este tipo de enseñanza y la insuficiente formación docente para abordar la educación socioemocional (O’Conner et al., 2017; Papadopouloua et al., 2014). Así, desde las educadoras de este estudio, educar en prosocialidad no es una tarea sencilla, porque se requiere de una educación capaz de combinar contextos de enseñanza formales e informales y de la participación de la familia. Al respecto, algunos autores destacan propuestas de enseñanza más centradas en el saber práctico, experiencial y de modelado (Henao, 2014); mientras que otros ponen énfasis en el desarrollo cognitivo, para favorecer el aprendizaje de la conciencia social y la empatía (O’Conner et al., 2017).

Desde estos hallazgos, parece prudente que la teoría del comportamiento prosocial, así como las políticas educativas y los servicios de apoyo profesional a los grupos familiares, tengan en cuenta los facilitadores y obstaculizadores de la enseñanza y aprendizaje de este comportamiento en la educación inicial, que las educadoras de este estudio identifican. Para Roche (1997), los comportamientos prosociales son facilitados por los hábitos, actitudes, rasgos de personalidad y por los contextos donde se vive o actúa. En contexto escolar, se requiere de un ambiente motivador, profesores(as) con una acti-tud favorable frente a la educación socioemocional, que fomenten relaciones de ayuda y solidaridad entre los(as) estudiantes (Dorantes, 2011) y las modelen (Durango, Soto y Yara, 2014).

También se requiere abordar activamente los factores obstaculizadores del trabajo docente para la educación en prosocialidad. El primer desafío es promover políticas educativas que le otorguen un mayor espacio a la educación socioemocional en las escuelas y al mismo tiempo, generen las condiciones estructurales para que los docentes ejerzan con el debido apoyo esta función. El segundo desafío se centra en la educación familiar, para tener padres más preparados para el desarrollo integral de sus hijos. Respecto de esto último, parece urgente aunar los saberes científicos y profesionales acerca de la crianza familiar, porque actualmente los padres ejercen esta tarea a partir de lo que múltiples fuentes de información le insuman, muchas veces sin sustento teórico e incluso contradictorias. La escuela podría ser una de las alternativas para la educación parental, pero para esto se requieren po-líticas que la agencien, preparen y doten de los recursos necesarios. 
También se requiere de nuevas investigaciones empíricas que aporten en metodologías y didácticas para la enseñanza de la prosocialidad en contexto educativo, sobre todo durante la educación inicial (Papadopouloua et al., 2014). Edo, Blanch y Anton (2016) proponen que el incremento en la cotidianidad de las conductas prosociales, disminuye conductas negativas en los estudiantes. En esta misma línea, Acar y Torquati (2015) aportan evidencia de cómo el promover en los infantes una relación respetuosa y cercana con la naturaleza es una buena estrategia para desarrollar y educar en prosocialidad durante la educación inicial.

Hemos presentado un modelo descriptivo-interpretativo que permite comprender cómo es que las educadoras implementan la enseñanza y aprendizaje de la prosocialidad en los párvulos y promueven su desarrollo. En este modelo destacan tres niveles epistemológicos de conocimiento subjetivo docente que interactúan y se encuentran interrelacionados para orientar la acción del profesor. En otros trabajos se ha visto que las TS de paradigma, epistemológicas u ontológicas, son representaciones subjetivas complejas que subordinan a otras TS y regulan de manera importante la acción docente (Cuadra-Martínez et al., 2017). Esto advierte de la complejidad del conocimiento profesional del profesor y de la necesidad de repensar sus procesos formativos con base en estos saberes.

No obstante, este trabajo presenta algunas limitaciones, la muestra podría ser mayor y con mayor heterogeneidad, por ejemplo, en cuanto a años de experiencia del profesorado estudiado. Un estudio que superara esta limitación permitiría conclusiones que cumplieran mejor la necesaria transferibilidad de un estudio de esta naturaleza. Considerar todo lo anterior, permitiría avanzar en contribuir al fortalecimiento de la formación profesional docente, a la tan anhelada calidad educativa y a la formación de estudiantes más solidarios y bondadosos.

\section{Referencias}

Acar, I. y Torquati, J. (2015). The power of nature: Developing prosocial behavior toward nature and peers through nature-based activities. Young Children, 116, 62-71.

Anl, G. (2019). Adaptation of the prosocial behavioral intentions scale for use with Turkish participants: Assessments of validity and reliability. Current Psychology, 38, 950-958.

https://doi.org/10.1007/s12144-019-00277-y

Arias, W. (2015). Conducta prosocial y psicología positiva. Avances en Psicología, 23(1), 37-47. https://doi.org/10.33539/avpsicol.2015.v23n1.169

Auné, S., Blum, D., Abal, F., Lozzia, G. y Horacio, F. (2014). La conducta prosocial: Estado actual de la investigación. Perspectivas en Psicología, 11(2), 21-33.

Campos, M., Chacc, I. y Gálvez, P. (2006). El juego como estrategia pedagógica: Una situación de interacción educativa (Trabajo Fin de Grado). Universidad de Chile.

Castro, P., Jiménez, V, Olivares, F., Rivas, D., Trujillo, E. y Cuadra-Martínez, D. (2019). Preschool teachers' subjective theories about family discipline in eight public and private schools in Chile. Psychology and Education, 56(1), 1-23.

Catalán, J. (2016). Hacia la formulación de una teoría general de las teorías subjetivas. Psicoperspectivas, 15(1), 29-41.https://doi.org/10.5027/psicoperspectivas-Vol15-Issue1-fulltext-739

Chinea, A. (2015). Altruismo, empatía, autoestima: ¿Son las personas más solidarias las que más se oponen a recibir ayuda? (Trabajo Fin de Grado). Universidad de La Laguna.

Correa, M. (2017). Aproximaciones epistemológicas y conceptuales de la conducta prosocial. Revista del Instituto de Estudios en Educación Universidad del Norte, 27, 1-21.

Cuadra-Martínez, D. (2016). Cambio representacional en los padres: Reconstrucción de teorías subjetivas de la prevención del consumo de drogas en los hijos e hijas. Revista Estudios Pedagógicos, 42(1), 283-298.

https://doi.org/10.4067/S0718-07052016000100018 
Cuadra-Martínez, D., Castro, P. J. y Juliá, M. T. (2018). Tres saberes en la formación profesional por competencias: Integración de teorías científicas, subjetivas y profesionales. Formación Universitaria, 11(5), 19-30. https://doi.org/10.4067/S0718-50062018000500019

Cuadra-Martínez, D., Castro, P. J., Vystrčilová, P. y Jancic Mogliacci, R. (2017). A review of research on teachers' subjective theories: Contributions to the study of teacher education. Psychology and Education, 54(3), 1-15.

Dorantes, G. (2011). Las competencias del docente y su relación con el desarrollo de conductas prosociales en los niños en edad preescolar (Trabajo Fin de Grado). Tecnológico de Monterrey.

Dunfiel, K. y Kuhlmeier, V. (2013). Classifying prosocial behavior: Children 's responses to instrumental need, emotional distress, and material desire. Child Development, 84(5), 1766-1776. https:/doi.org/10.1111/cdev.12075

Durango, M., Soto, S. y Yara, F. (2014). Prosocialidad y convivencia escolar en niños y niñas en edades entre 6 y 9 años de la institución educativa Juan Echeverry Abad Itagüí, Antioquia (Trabajo Fin de Grado). Universidad Cooperativa de Colombia.

Edo, M., Blanch, S. y Anton, M. (2016). El juego en la primera infancia. Octaedro.

Eisenberg, N., Eggum, N. y Di Giunta, L. (2010). Empaty-related responding: Associations with prosocial behavior, aggression, and intergroup relations. Social Issues and Policy Review, 4, 143-180.

https://doi.org/10.1111/j.1751-2409.2010.01020.x

Flick, U. (2014). An introduction to qualitative research. Sage.

Garaigordobil, M. (2014). Conducta prosocial: El papel de la cultura, la familia, la escuela y la personalidad. Revista Mexicana de Investigación en Psicología, 6(2), 146-157.

García, B. (2010). Razonamiento y emoción moral, su vinculación con la conducta pro-social en la etapa preescolar. Revista Mexicana de Psicología Educativa, 1(1), 21-30.

Henao, P. (2014). Interacciones ritualizadas entre niños y niñas de 5 a 6 años de edad del nivel de preescolar en la institución educativa Manuel José Cayzedo. Un estudio etnográfico (Trabajo Fin de grado). Universidad de San Buenaventura.

Hepach, R., Vaish, A., Grossmann, T. y Tomasello, M. (2016). Young children want to see others get the help they need. Child Development, 87(6), 1703-1714. https://doi.org/10.1111/cdev.12633

Hollingsworth, H. L. y Winter, M. K. (2013). Teacher beliefs and practices relating to development in preschool: Importance placed on social-emotional behaviors and skills. Early Child Development and Care, 183(12), 1758-1781. https://doi.org/10.1080/03004430.2012.759567

Inglés, C. J., Martínez-González, A. y García-Fernández, J. M. (2013). Conducta prosocial y estrategias de aprendizaje en una muestra de estudiantes españoles de educación secundaria obligatoria. European Journal of Education and Psychology, 6(1), 33-53. https://doi.org/10.30552/ejep.v6i1.88

Kowalski, K., Pretti-Frontczak, K. y Johnson, L. (2001). Preschool teachers' beliefs concerning the importance of various developmental skills and abilities. Journal of Research in Childhood Education, 16, 5-14. https://doi.org/10.1080/02568540109594970

Kelchtermans, G. (2009). Who I am in how I teach is the message: Self-understanding, vulnerability and reflection. Teachers and Teaching, 15(2), 257-272. https://doi.org/10.1080/13540600902875332

Lay, J. y Hoppmann, C. (2015). Altruism and prosocial behavior. Encyclopedia of Geropsychology, 69, 1-9. https://doi.org/10.1007/978-981-287-080-3_69-1

Lería, F., Salgado, J. y Sasso, P. (2018). Sentido subjetivo de educadoras de párvulos en el uso e impacto del silencio en el aprendizaje. REICE. Revista Iberoamericana sobre Calidad, Eficacia y Cambio en Educación, 16(2), 79-93. https://doi.org/10.15366/reice2018.16.2.005

Li, Y., Coplan, R., Archbell, K., Bullock, A. y Chen, L. (2016). Chinese kindergarten teachers' beliefs about young children's classroom social behavior. Early Childhood Research Quarterly, 36, 122-132.

https://doi.org/10.1016/j.ecresq.2015.10.008 
McLaughlin, T., Aspden, K. y Clarke, L. (2017). How do teachers support children's social emotional competence? Strategies for teachers. Early Childhood Folio, 21(2), 21-26. https://doi.org/10.18296/ecf.0041

O’Conner, R., De Feyter, J., Carr, A., Luo, J. L. y Romm, H. (2017). A review of the literature on social and emotional learning for students ages 3-8: Characteristics of effective social and emotional learning programs. Regional Educational Laboratory Mid-Atlantic.

Papadopouloua, K., Tsermidoua, L., Dimitrakakib, C., Agapidakib, E., Oikonomidoub, D., Petanidoub, D., Tountasb, Y. y Giannakopoulosb, G. (2014). A qualitative study of early childhood educators' beliefs and practices regarding children's socioemotional development. Early Child Development and Care, 184(12), 1843-1860. https://doi.org/10.1080/03004430.2014.889693

Redondo, J., Rueda, S. y Amado, C. (2013). Conducta prosocial: Una alternativa a las conductas agresivas. Revista Investigium IRE: Ciencias Sociales y Humanas, 4(1), 234-247.

Rimm-Kaufman, S. E., Paro, K. M., Downer, J. y Pianta, R. C. (2005). The contribution of classroom setting and quality of instruction to children's behavior in the kindergarten classroom. The Elementary School Journal, 105, 377-394. https://doi.org/10.1086/429948

Roche, R. (1997). El servicio a la comunidad como aprendizaje escolar. Ministerio de Cultura y Educación Argentino.

Sánchez-Queija, I., Oliva, A. y Parra. A. (2006). Empatía y conducta prosocial durante la adolescencia. Revista de Psicología Social, 21(3), 259-271. https://doi.org/10.1174/021347406778538230

Shirin, A. (2020). Determining the relationship between academic achievement and prosocial behavior of secondary school students in Dhaka City. International Journal of Research and Reviews in Education, 6, 6-15. https://doi.org/10.33500/ijrre.2020.06.002

Sims, M., Davis, E., Davies, B., Nicholson, J. M., Harrison, L., Herrman, H., Waters, E., Marshall, B., Cook, K. y Priest, N. (2012). Mental health promotion in childcare centres: Childcare educators' understanding of child and parental mental health. Advances in Mental Health, 10(2), 138-148. https://doi.org/10.5172/jamh.2011.10.2.138

Son, D. y Padilla-Walker, L. (2019). Happy helpers: A multidimensional and mixed-method approach to prosocial behavior and its effects on friendship quality, mental health, and well-being during adolescence. Journal of Hajavippiness Studies, 21,1-19. https://doi.org/10.1007/s10902-019-00154-2

Stake, R. (1995). Investigación con estudio de casos. Morata.

Strauss, A. y Corbin, J. (2002). Bases de la investigación cualitativa. Técnicas y procedimientos para desarrollar la teoría fundamentada. Editorial Universidad de Antioquia.

Taylor, S. J. y Bogdan, R. (2000). Introducción a los métodos cualitativos de investigación. Paidós.

Tomasello, M. (2010). ¿Por qué cooperamos? Katz.

Wang, J., Elicker, J., McMullen, M. y Mao, S. (2008). Chinese and American preschool teachers' beliefs about early childhood curriculum. Early Child Development and Care, 178(3), 227-249. https://doi.org/10.1080/03004430600722671

Wen, X., Elicker, J. y McMullen, M. B. (2011). Early childhood teacher's curriculum beliefs: Are they consistent with observed classroom practices? Early Education and Development, 22(6), 945-969.

https://doi.org/10.1080/10409289.2010.507495

\section{Breve CV de los/as autores/as}

\section{Ginella Cicardini}

Psicóloga y Licenciada en Psicología en la Universidad de Atacama, Chile. Se desempeña como Consejera Familiar en el Programa Preventivo Focalizado Horizonte, de la Fundación Instituto de Educación Popular. Posee experiencia profesional en educación parental e infancia, específicamente en el fortalecimiento de las competencias de cuidado y crianza de familias y/o adultos significativos de los niños, niñas, adoles- 
centes que han sido vulnerados en sus derechos. Sus intereses investigativos son la subjetividad y educación, la formación profesional y el desarrollo, enseñanza y aprendizaje del comportamiento prosocial en la infancia y los grupos familiares. Email: ginella.cicardini@gmail.com

ORCID: https://orcid.org/0000-0002-1775-8198

\section{Macarena Martínez}

Psicóloga y Licenciada en psicología en la Universidad de Atacama, Chile. Se desempeña como Psicóloga Escolar en el Liceo Polivalente El Palomar, Atacama, Chile, en donde planifica e implementa programas de intervención para estudiantes secundarios con alta vulnerabilidad social, focalizándose en la inclusión educativa, las necesidades educativas especiales, el desarrollo psicosocial, socioemocional y cognitivo de estudiantes y la convivencia escolar. Ocupa el cargo de supervisora en Proyecto Aplauso Chile. Sus intereses de investigación son la subjetividad y educación, las necesidades educativas especiales, el clima escolar y el comportamiento prosocial en contexto educativo. Email: macarenamartinezduran1996@gmail.com

ORCID: https://orcid.org/0000-0002-8790-6926

\section{Karen Ramírez}

Psicóloga y Licenciada en Psicología en la Universidad de Atacama, Chile. Se desempeña como Consejera Familiar en el Programa Preventivo Focalizado Horizonte, de la Fundación Instituto de Educación Popular. Posee experiencia profesional en educación parental e infancia, específicamente en el fortalecimiento de las competencias de cuidado y crianza de familias y/o adultos significativos de los niños, niñas, adolescentes que han sido vulnerados en sus derechos, además de formación en prevención del Abuso Sexual Infantil y en la Detección temprana, Prevención universal y ambiental del consumo de sustancias y drogas. Sus intereses investigativos son la subjetividad y educación, la formación profesional y el comportamiento prosocial. Email: karen.ramirez.ag@gmail.com

ORCID ID: https://orcid.org/0000-0002-2803-2149

\section{David Cuadra-Martínez}

Psicólogo, Magíster en Psicología. Académico de asignaturas de pregrado del Departamento de Psicología de la Facultad de Humanidades y Educación de la Universidad de Atacama, Chile. Profesor del programa de magíster en Psicología de la Universidad de Atacama. Integrante del Comité evaluador de la Sub Dirección de Capital Humano de la Agencia Nacional de Investigación y Desarrollo (Conicyt). Actualmente es investigador responsable del proyecto Fondecyt Regular 1201084, cuyo objetivo es comprender la construcción de la identidad profesional docente durante la formación inicial. Sus líneas de investigación son la formación profesional, la identidad profesional, la enseñanza y aprendizaje y el comportamiento prosocial. Email: david.cuadra@uda.cl

ORCID: http://orcid.org/0000-0002-0810-2795

\section{Pablo J. Castro-Carrasco}

Profesor titular del Departamento de Psicología de la Universidad de La Serena (Chile). Castro es Licenciado en Psicología de la Universidad de La Serena (Chile); Doctor en Psicología de la Pontificia Universidad Católica de Chile. Ha realizado estancias de investigación doctoral y posdoctoral en la Universidad de Ciencias Aplicadas Alice Salomon de Berlín, Alemania, la Universidad de La Laguna, España, y la Universidad de Leiden, Paises Bajos. Sus áreas de investigación son teorías subjetivas, tiempo escolar, identidad profesional docente y convivencia escolar. Ha publicado en revistas cientìficas nacionales e internacionales. Email: pablocastro@userena.cl

ORCID: https://orcid.org/0000-0002-8640-5820 


\section{Nidia Slomp}

Profesora de Estado en Inglés (Universidad de La Serena, Chile). Académica de la Universidad de La Serena de la Carrera de Pedagogía en inglés a cargo de las asignaturas de Didáctica y Prácticas en Contexto Profesional. Licenciada en Educación por la Universidad de La Serena (Chile). Master en Enseñanza de Inglés (Master in ELT. English Language Teaching) University of Southampton, UK. Phd(c) en Lingüística Aplicada, University of Southampton. Ha investigado sobre teorías subjetivas de docentes y acerca de la instrucción en base al "input" (processing instruction) en la adquisicion de un segundo idioma. Email: nslomp@userena.cl

ORCID: https://orcid.org/0000-0001-8832-8542 